4 European Centre for Disease Prevention and Control. Framework Action Plan to Fight Tuberculosis in the European Union. Stockholm, ECDC, 2008. Available from: http://ecdc.europa.eu/en/publications/Publications/0803_ SPR_TB_Action_plan.pdf

5 Walker TM, Lalor MK, Broda A, et al. Assessment of Mycobacterium tuberculosis transmission in Oxfordshire, UK, 2007-12, with whole pathogen genome sequences: an observational study. Lancet Respir Med 2014; 2: 285-292.

6 Sanchez-Padilla E, Merker M, Beckert P, et al. Detection of drug-resistant tuberculosis by Xpert MTB/RIF in Swaziland. N Engl J Med. 2015; 372: 1181-1182.

7 Starks AM, Avilés E, Cirillo DM, et al. Collaborative effort for a centralized worldwide tuberculosis relational sequencing data platform. Clin Infect Dis 2015; 61: Suppl. 3, 141-146.

8 Sotgiu G, Mauch V, Migliori GB, et al. Evidence-based, agreed-upon health priorities to remedy the tuberculosis patient's economic disaster. Eur Respir J 2014; 43: 1563-1566.

9 Diel R, Loddenkemper R, Zellweger JP, et al. Old ideas to innovate tuberculosis control: preventive treatment to achieve elimination. Eur Respir J 2013; 42: 785-801.

\title{
Linezolid in drug-resistant tuberculosis: haste makes waste
}

To the Editor:

Hughes et al. [1] should be commended for their interesting short study on evaluating the efficacy of linezolid in drug-resistant tuberculosis (TB). However, there are some points of concern that need to be discussed. A recent systematic review and meta-analysis cautions the use of linezolid in multidrug-resistant (MDR) TB because of the frequency and severity of adverse events (i.e. peripheral and optic neuropathy, myelosuppression and gastrointestinal disorders), despite its excellent efficacy. This robust study recommends the use of linezolid in only severe and difficult-to-treat cases but under strict supervision [2].

The retrospective study included patients with MDR-TB, extensively drug-resistant (XDR) TB and pre-XDR-TB (this terminology, although not unanimously recognised everywhere, has been used by several clinicians and defined as MDR plus resistance to either a fluoroquinolone or an injectable agent) from resource constraint areas with high burden of TB. Under such a scenario it would have been prudent to start standardised treatment regimens as per the World Health Organization (WHO) guidelines [3], rather than individualised regimens without the feasibility of individual drug susceptibility test (DST) for each of the second-line anti-TB drugs. It is clear from the WHO recommendations that the first choice is to use individualised regimen tailored by DST, the second choice of standardised regimens being determined by the impossibility to perform systematic DST in all MDR/XDR-TB strains. However, with the gradually increasing number of advanced DST laboratories, the individualised regimens are bound, in the future, to replace the standardised regimens in these high burden countries. This in turn may curb the emergence of drug-resistant $\mathrm{TB}$, which mostly results from improper and non-standardised treatment regimens, often prescribed by practitioners in these high burden areas in the absence of DST for individual drugs [4]. The authors recommend early use of linezolid containing individualised regimen on initial detection of second-line drug resistance to achieve better treatment outcomes. However, we would like to mention here that linezolid containing standardised treatment regimen for XDR-TB has already been included in the national treatment guideline of India (one of the sites included in the study) as per the recommendations of $\mathrm{WHO}$, and it does not recommend the use of linezolid in MDR- or pre-XDR-TB [5].

Secondly, nothing has been mentioned about the other drugs included in the treatment regimens. The findings could have been more valid if they were compared with a control group treated with non-linezolid containing regimens. Since linezolid belongs to the group-5 of anti-TB drugs, as per the WHO guidelines, whenever a drug is included from this group it should be accompanied by two or more other drugs from this group [3]. This is particularly important to preserve the efficacy of these relatively newer anti-TB drugs.

While labelled use of linezolid is restricted to 28 days, with dosage of $600 \mathrm{mg}$ twice daily, drug-resistant TB requires a much longer treatment duration, often for $\geqslant 2$ years and, therefore, carries an increased likelihood of severe adverse events, more so if the patient is receiving concurrent antiretroviral therapy [6]. This may lead to drug intolerance and withdrawal of linezolid from the regimen. A recent prospective randomised trial on XDR-TB patients has demonstrated the efficacy of a reduced linezolid dose 
(300-600 mg per day). However, it recommends that the safety profile of linezolid does not warrant its use in cases where there are other, safer, alternatives [7]. Furthermore, use of linezolid in improper dosage and duration have been associated with increased risk of acquired drug resistance and adverse effects/outcome may be more in those patients of XDR-TB infected with linezolid resistant strains when treated with regimens containing linezolid [8].

We have already lost the two most important first-line drugs, i.e. rifampicin and isoniazid to MDR-TB, and the two most important second-line drugs, i.e. fluoroquinolone and injectable anti-TB drugs, to $\mathrm{XDR}-\mathrm{TB}$ and now it would not be wise to lose one of the key drugs in the treatment of XDR-TB, i.e. linezolid to the imminent "resistance beyond XDR-TB" [9]. Several clinicians refer to this dreaded condition as total drug resistance, although this terminology has not yet been recognised by WHO. Hence, at this juncture, it would be sensible to restrict the use of linezolid in XDR-TB and complicated drugresistant TB cases only, as per the WHO guidelines, rather than in all cases of drug-resistant TB. It is well known that treatment outcomes, even under optimal conditions, are relatively poor for MDR-TB and are even worse for XDR-TB [10]. Treatment options for these XDR-TB patients are extremely limited and often rely on a set of drugs with poorly established efficacy and severe adverse events profile. Therefore, the drugs in the armamentarium for XDR-TB should be used judiciously and with utmost care, lest we run out of ammunition in the battle field of XDR-TB.

@ERSpublications

The use of linezolid should be preserved for XDR-TB and complicated drug-resistant-TB only http://ow.ly/Tzg66

Sourin Bhuniya, Prasanta Raghab Mohapatra, Manoj Kumar Panigrahi, Priyadarshini Behera and Gourhari Pradhan Dept Pulmonary Medicine, All India Institute of Medical Sciences (AIIMS), Bhubaneswar, India.

Correspondence: Sourin Bhuniya, Dept of Pulmonary Medicine, All India Institute of Medical Sciences (AIIMS) Bhubaneswar, Sijua, Dumduma, Bhubaneswar - 751019, India. E-mail: sbhuniya@hotmail.com

Received: July 192015 | Accepted: Aug 042015

Conflict of interest: None declared.

\section{References}

1 Hughes J, Isaakidis P, Andries A, et al. Linezolid for multidrug-resistant tuberculosis in HIV-infected and uninfected patients . Eur Respir J 2015; 46: 271-274.

2 Sotgiu G, Centis R, D'Ambrosio L, et al. Efficacy, safety and tolerability of Linezolid containing regimens in treating MDR-TB and XDR-TB: systematic review and meta-analysis. Eur Respir J 2012; 40: 1430-1442.

3 World Health Organization. Companion handbook to the WHO guidelines for programmatic management of drug resistant tuberculosis. WHO/HTM/TB/2014.11. Geneva, WHO, 2014. Available from: http://www.who.int/tb/ publications/pmdt_companionhandbook/en/

4 Caminero JA. Multidrug-resistant tuberculosis: epidemiology, risk factors and case finding. Int J Tuberc Lung Dis 2010; 14: 382-390.

5 Revised National Tuberculosis Control Program. Guidelines on the programmatic management of drug resistant tuberculosis (PMDT) in India. Central TB Division Ministry of Health and Family Welfare, New Delhi, 2012. Available from: http://www.tbcindia.nic.in/pdfs/Guidelines\%20for\%20PMDT\%20in\%20India\%20-\%20May\%202012.pdf

6 Cox H, Ford N. Linezolid for the treatment of complicated drug-resistant tuberculosis: a systematic review and meta-analysis. Int J Tuberc Lung Dis 2012; 16: 447-454.

7 Migliori GB, Eker B, Richardson MD, et al. A retrospective TBNET assessment of linezolid safety, tolerability and efficacy in multidrug-resistant tuberculosis. Eur Respir J 2009; 34: 387-393.

8 Zhang L, Pang Y, Yu X, et al. Linezolid in the treatment of extensively drug-resistant tuberculosis. Infection 2014; 42: 705-711.

9 Migliori GB, Sotgiu G, Gandhi NR, et al. Drug resistance beyond extensively drug-resistant tuberculosis: individual patient data meta-analysis. Eur Respir J 2013; 42: 169-179.

10 Jacobson KR, Tierney DB, Jeon CY, et al. Treatment outcomes among patients with extensively drug-resistant tuberculosis: systematic review and meta-analysis. Clin Infect Dis 2010; 51: 6-14.

Eur Respir J 2015; 46: 1843-1844 | DOI: 10.1183/13993003.01162-2015 | Copyright @eES 2015

From the authors:

We thank Bhuniya and colleagues for highlighting these common concerns regarding the use of linezolid in drug-resistant tuberculosis (DR-TB). We confirm that patients in our cohort were initially started on treatment with standardised regimens following diagnosis of rifampicin and isoniazid resistance (multidrug-resistant tuberculosis (MDR-TB)). We did have access to drug susceptibility testing (DST) for 